Am J Perinatol. 2018 July ; 35(8): 758-763. doi:10.1055/s-0037-1615792.

\title{
Outcomes of Medically Indicated Preterm Births differ by Indication
}

\author{
Michelle J. WANG, AB ${ }^{1}$, Spencer KUPER, MD ${ }^{1}$, Robin STEELE, MD, MPH ${ }^{1}$, Rachel SIEVERT, \\ MD ${ }^{1}$, Alan T. TITA, MD, PhD ${ }^{1}$, Lorie HARPER, MD, MSCI ${ }^{1}$ \\ ${ }^{1}$ Division of Maternal Fetal Medicine. Department of Obstetrics and Gynecology, Center for \\ Women's Reproductive Health at the University of Alabama at Birmingham, Birmingham, AL
}

\begin{abstract}
Objective-We aim to examine whether outcomes of preterm birth (PTB) are further modified by the indication for delivery.

Study Design-We performed a retrospective cohort study of all singletons delivered at 23-34 weeks from 2011-2014. Women were classified by their primary indication for delivery: maternal (preeclampsia) or fetal/obstetric (growth restriction, non-reassuring fetal status, vaginal bleeding). The primary neonatal outcome was a composite of neonatal death, cord $\mathrm{pH}<7$ or base excess $<$ -12 5-minute Apgar 3 , CPR during resuscitation, culture-proven sepsis, intraventricular hemorrhage, and necrotizing enterocolitis. Secondary outcomes included the individual components of the primary outcome. Groups were compared using Student's t-test and chisquared tests. Logistic regression was used to adjust for confounding variables.
\end{abstract}

Results-Of 528 women, 395 (74.8\%) were delivered for maternal and 133 (25.2\%) for fetal/ obstetric indications. Compared to those delivered for a maternal indication, those with a fetal/ obstetric indication for delivery had an increased risk of the composite neonatal outcome (AOR $1.9,95 \%$ CI 1.13-3.21) and acidemia at birth (AOR 4.2, 95\%CI 1.89-9.55).

Conclusion-Preterm infants delivered for fetal/obstetric indications have worsened outcomes compared to those delivered for maternal indications. Additional research is needed to further tailor counseling specific to the indication for delivery.

\section{Keywords}

Preterm birth; Neonatal morbidity; Indication for preterm birth; Neonatal outcomes

\section{Introduction}

Indicated preterm births represent nearly $35 \%$ of preterm births. ${ }^{1}$ The most common indications for indicated preterm deliveries are fetal (non-reassuring fetal status) and hypertensive disorders. ${ }^{2}$

Corresponding author: Michelle J. Wang, The University of Alabama at Birmingham, Department of Obstetrics and Gynecology, Address: $17006^{\text {th }}$ Ave South Ste 10270, Birmingham, AL 35233, Phone: 205-934-5631 Fax: 205-975-4375 mjoyw@uab.edu. 
The primary determinant of neonatal outcomes is gestational age; however, race, fetal gender, and administration of antenatal corticosteroids are all important factors in survival and intact survival. ${ }^{3}$ Recent research is beginning to show that the indication for the delivery is also an important factor in counseling for the sake of both neonatal outcomes and maternal outcomes. ${ }^{4,5}$ The majority of previous studies compare the neonatal outcomes of spontaneous preterm deliveries with the outcomes of all medically indicated preterm births. Few have examined the differences of outcomes among the range of medical indications for preterm deliveries; in studies that have examined among various indications, only intrauterine growth restriction (IUGR) was found to have a significant increased neonatal risk. ${ }^{6,7}$ Therefore, the current study presents an important opportunity to expand our understanding of the outcomes among other medical indications for preterm deliveries and personalize care for individual mothers and their preterm newborns.

We aim to examine the impact of indication for preterm delivery (maternal, fetal or obstetric) on immediate neonatal outcomes. Given that previous studies demonstrate an increased rate of morbidities associated with IUGR for preterm delivery, we hypothesize that immediate neonatal outcomes will vary by indication for delivery, specifically that those delivered for fetal or obstetric indications will have a worse prognosis compared to those delivered for other indications.

\section{Materials and Methods}

This was a retrospective cohort study of all preterm singletons with an indicated delivery from January 1, 2011 to December 31, 2014. Institutional review board approval was obtained from the University of Alabama at Birmingham.

All women delivered 23-34 weeks at a single institution from 2011-2014. At our institution, maternal-fetal medicine faculty and fellows provide 24-hour coverage of labor and delivery 7 days per week. Trained chart abstractors completed standardized chart abstraction forms using a secure database. Information that was obtained include: maternal demographics, maternal medical and obstetric history, antenatal course, and pregnancy outcomes, and primary indications for delivery. In cases where multiple indications were listed, the primary indication for delivery was selected based on physician documentation. Subjects were excluded for delivery prior to 23 weeks, lack of fetal monitoring (or no intent to resuscitate), fetal anomalies, intrauterine fetal demise, spontaneous labor (including preterm premature rupture of membranes) suspected morbidly adherent placenta, maternal cardiac disease or testing HIV-positive.

Women were classified by their primary indication for delivery: maternal (preeclampsia) or fetal/obstetric. The majority of fetal and obstetric indications were non-reassuring fetal status, placental abruption, placenta previa with bleeding, and fetal growth restriction

The primary neonatal outcome was a composite of neonatal death, cord $\mathrm{pH}<7$ or base excess <-12, 5-minute Apgar 3 , CPR during resuscitation, culture-proven sepsis, grade IIIIV intraventricular hemorrhage, and necrotizing enterocolitis. Secondary outcomes included the individual components of the primary outcome. These outcomes were chosen as they are 
associated with significant risks of neonatal mortality or long-standing neonatal morbidities, including hypoxic ischemic encephalopathy. ${ }^{8-17}$

The exposure groups were compared with descriptive and bivariate statistics using Student's t-test for continuous variables and chi-squared test for categorical variables. Potentially confounding variables of the exposure-outcome association were identified in the stratified analyses. Multivariable logistic regression models for the primary and secondary outcomes were then developed to estimate the effect of indication for delivery on immediate neonatal outcomes, using maternal indications as the reference group. Clinically relevant covariates for initial inclusion in multivariable statistical models were selected using results of the stratified analyses, and factors were removed in a backward stepwise fashion, based on significant changes in the exposure adjusted odds ratio or significant differences between hierarchical models using the likelihood ratio test. Confounding factors considered included antenatal corticosteroids steroids, gestational age at delivery, maternal body mass index, race, sex of the neonate, intended mode of delivery, and parity. All analyses were completed using STATA SE, version 13 (College Station, TX).

\section{Results}

Of 1,352 identified with a singleton preterm birth at 23-34 weeks, 824 were excluded (703 excluded for spontaneous labor, 65 excluded for fetus with anomalies, 19 excluded for maternal cardiac disease, 17 excluded for lack of fetal monitoring, 9 for suspected morbidly adherent placenta, 5 excluded for rare maternal indications other than preeclampsia, 3 excluded for being tested HIV positive, 2 excluded for outside the gestational age range, and 1 for unclear indication for delivery). Of the 528 women eligible, 395 (74.8\%) had a maternal indication as their primary indication for delivery, $133(25.2 \%)$ had a fetal/obstetric indication (Figure 1). For those in the fetal/obstetric indication category, $99(74.4 \%)$ had non-reassuring fetal status, 12 (9.0\%) had placental abruption, 7 (5.3\%) had vaginal bleeding, $6(5.4 \%)$ were intrauterine growth restricted (IUGR) with reversal of end-diastolic flow, 5 (4.5\%) had IUGR with absent end-diastolic flow, 2 (1.5\%) had oligohydramnios, 1 $(0.9 \%)$ had IUGR with normal umbilical artery Doppler, and $1(0.9 \%)$ had suspected isoimmunization. Of those included with a maternal indication as their primary indication, $395(100 \%)$ had pre-eclampsia, eclampsia or HELLP.

Maternal characteristics according to primary indication for delivery are shown in Table 1. Exposure groups were not significantly different with regards to maternal age, gestational age at delivery, marital status, race, previous preterm pregnancies diabetes, and renal disease. Women with a maternal indication for delivery were more likely to be nulliparous, have chronic hypertension, and have a higher body mass index than the obstetric or fetal groups. Those with a maternal indication were less likely to have had a prior vaginal delivery, smoke, and report alcohol or drug use.

Obstetric characteristics are shown in Table 2. Preeclampsia was diagnosed in all of the maternal indication group, but was also diagnosed in over 35.3\% of the fetal/obstetric indication group $(\mathrm{p}<0.01)$. Estimated fetal weight was lowest and the diagnosis of fetal growth restriction was most common in the fetal/obstetric indications group $(\mathrm{p}<0.01)$, as 
were critically abnormal Dopplers in those diagnosed with fetal growth restriction $(\mathrm{p}<0.01)$. Patients in the maternal indications group were most likely to complete a course of antenatal corticosteroids $(\mathrm{p}<0.01)$. Gestational age and birth weight were significantly different between groups, with the fetal indication group having the earliest gestational age and lowest birth weight.

Neonatal outcomes according to primary indication are displayed in Table 3. Infants in the fetal/obstetric indication group had a higher incidence of the neonatal composite outcome compared to the maternal indication groups $(\mathrm{p}<0.01$ ). After adjusting for antenatal corticosteroid use, gestational age at delivery and maternal BMI, neonates delivered for fetal/obstetric indications had a significantly higher odds of the composite neonatal outcome compared to those delivered for maternal indications (AOR 1.9, 95\% CI 1.14-3.21).

We then examined components of the primary neonatal outcome individually. An increase in the risk of neonatal death in the fetal/obstetric indication group did not remain statistically significant compared to the maternal indication group after adjusting for gestational age at delivery and antenatal corticosteroids. Those delivered for fetal/obstetric indications were at significant increased risk of having an acidemic cord blood gas (AOR 4.2, 95\% CI 1.899.55). The remaining components of the primary outcome (intraventricular hemorrhage, culture-proven sepsis, necrotizing enterocolitis, 5-minute Apgar $\mathfrak{3}$, and CPR in the delivery room) were not significantly increased in the fetal indication or obstetric indication groups compared to those delivered for maternal indications after adjusting for important confounding factors.

\section{Discussion}

In this cohort of women undergoing indicated preterm deliveries between 23-34 weeks, fetal and obstetric indications for delivery were associated with worsened immediate neonatal outcomes compared to maternal indications.

Medically indicated preterm births have been an increasing area of study in recent years, with several large population-based studies demonstrating that indicated preterm birth are associated with higher morbidity and mortality risk when compared to spontaneous preterm labor. ${ }^{4-7,18-20}$ In a multicenter prospective study with a comparable total sample size, Garite et al. found that of all medically indicated preterm deliveries in their population, only suspected IUGR infants had worsened outcomes. ${ }^{7}$ Our study findings suggest that infants delivered due to any fetal or obstetric indication may have an increased risk for severe morbidities, not just those that are growth-restricted.

Prior studies examining neonatal outcomes in preterm deliveries have focused primarily on differences in spontaneous versus medically indicated preterm deliveries as an overarching category. Recent studies have shown, however, that there is a broad spectrum of heterogeneity of clinical subtypes of medically indicated preterm birth, which are each associated with different risk factors and causal factors. ${ }^{21}$ As this variance in underlying causes potentially has a diverse influence on delivery outcomes, those previous studies contrasting outcomes of all medically indicated preterm births to spontaneous births may 
have drawn overly broad comparisons. Our focus on differences among medically indicated preterm deliveries allows for more detailed counseling of this patient population.

Previous studies have mostly used large existing national databases which provide a large sample size to examine rare outcomes. However, due to known underreporting of medical diagnoses and obstetric complications, these databases are limited by misclassification biases. $^{22-3}$ Our study with data collected from medical records by trained abstractors limits misclassification bias; additionally, due to the high risk nature of this cohort, we maintained the sample size required to examine rare neonatal outcomes. Finally, we selected as our primary neonatal outcome a composite of significant adverse events that are strongly associated with significant neonatal morbidity and mortality. ${ }^{8-17}$

We acknowledge some study limitations. First, although we attempted to control for the known differences between groups, residual confounding may persist. Furthermore, only preeclampsia was studied as a maternal indication for delivery due to the rarity of other indications. Some patients may have qualified for more than one single indication group; however, to simplify our analysis, we chose to focus on what we determined through the chart extraction process to be the "primary" indication for delivery. While we recognize that multiple indications may exist, we selected the indication listed as the delivering physician as primary. For example, a patient expectantly managed for preeclampsia who was delivered for non-reassuring fetal status would have been classified as obstetric. Stout et al have demonstrated reasonable inter-observer agreement with preterm birth classification. ${ }^{24}$ While patients could have been misclassified (biasing our results to the null), the detailed patientlevel data available likely enabled us to more accurately classify patients than large birth certificate databases allow.

In sum, the indication for medically indicated preterm births appears to have a significant impact on important immediate neonatal outcomes. However, existing evidence-based counseling lacks concise and differentiated strategies for treating various types of medically indicated preterm deliveries. With personalized medicine at frontier of patient care as providers seek to utilize the increasing breadth of specific diagnostic information to create individualized interventions, the information gathered in this study presents important initial preterm indication-specific data to help refine obstetric counseling. Further research is thus needed to examine the outcomes of different interventions on specific indications to allow physicians to tailor counseling to the indication for delivery in addition to current measures of gestational age and fetal weight.

\section{Acknowledgments}

Michelle J. Wang was supported by the National Center for Advancing Translational Sciences of the National Institutes of Health under Award Number TL1TR001418. The content is solely the responsibility of the authors and does not necessarily represent the official views of the National Institutes of Health.

Dr. Harper is supported by K12HD001258-13, PI WW Andrews, which partially supports this work. 


\section{References}

1. Loftin RW, Habli M, Snyder CC, Cormier CM, Lewis DF, \& DeFranco EA Late Preterm Birth. Reviews in Obstetrics and Gynecology 2010;3:10-19. [PubMed: 20508778]

2. Meis Paul J. et al. The Preterm Prediction Study: Risk Factors for Indicated Preterm Births. American Journal of Obstetrics and Gynecology 1998;178: 562-67. [PubMed: 9539527]

3. NICHD Neonatal Research Network (NRN): Extremely Preterm Birth Outcome Data. https:// www.nichd.nih.gov/about/org/der/branches/ppb/programs/epbo/pages/epbo_case.aspx. 11 30, 2012 Accessed August 27, 2016.

4. Kamath-Rayne Beena D., Defranco Emily A., Chung Ethan, and Chen Aimin. Subtypes of Preterm Birth and the Risk of Postneonatal Death. The Journal of Pediatrics 2013;162:28-34 [PubMed: 22878113]

5. Reddy Uma M., Ko Chia-Wen, Raju Tonse N. K., and Willinger Marian. Delivery Indications at Late-Preterm Gestations and Infant Mortality Rates in the United States. Obstetrical \& Gynecological Survey 2009;64:780-81.

6. Garite TJ, Clark RH, Thorp JA Intrauterine growth restriction increases morbidity and mortality among premature neonates. Am J Obstet Gynecol. 2004; 191: 481-487 [PubMed: 15343225]

7. Garite TJ, Combs CA, Maurel K, et al. A multicenter prospective study of neonatal outcomes at less than 32 weeks associated with indications for maternal admission and delivery. Am J Obstet Gynecol 2017; in press.

8. Lane I Neonatal Outcome of Infants with Significant Umbilical Artery Acidosis at Birth. Obstetrics \& Gynecology 2003;101: 72S-73S

9. Holmqvist P, Pleven H and Svenningsen NW (1988), Vaginally Born Low-Risk Preterm Infants: Fetal Acidosis and Outcome at 6 Years of Age. Acta Pædiatrica 1988; 77: 638-641

10. Chalak LF, Rollins N, Morriss MC, Brion LP, Heyne R, Sánchez PJ. Perinatal Acidosis and Hypoxic-Ischemic Encephalopathy in Preterm Infants of 33 to 35 Weeks' Gestation. The Journal of Pediatrics. 2012;160(3):388-394. [PubMed: 22033298]

11. Lee Henry Chong, Subeh Mohammad, and Gould Jeffrey B. "Low Apgar Score and Mortality in Extremely Preterm Neonates Born in the United States.” Acta Paediatrica 2010; 99: 1785-1789. [PubMed: 20626363]

12. Laptook AR, Shankaran S, Ambalavanan N, et al. Prediction of Early Childhood Outcome of Term Infants using Apgar Scores at 10 Minutes following Hypoxic-Ischemic Encephalopathy. Pediatrics. 2009;124(6):1619. [PubMed: 19948631]

13. Wyckoff MH, Salhab WA, Heyne RJ, Kendrick DE, Stoll BJ, Laptook AR. Outcome of Extremely Low Birth Weight Infants Who Received Delivery Room Cardiopulmonary Resuscitation. The Journal of Pediatrics 2012;160(2):239-244 [PubMed: 21930284]

14. Handley SC, Sun Y, Wyckoff MH, Lee HC. Outcomes of Extremely Preterm Infants after Delivery Room Cardiopulmonary Resuscitation in a Population-Based Cohort. Journal of Perinatology 2015; 35:379-383 [PubMed: 25521563]

15. Farris Reid W. D., Weiss Noel S., and Zimmerman Jerry J. Functional Outcomes in Pediatric Severe Sepsis. Pediatric Critical Care Medicine 2013;14:835-42. [PubMed: 24108117]

16. Bolisetty S, Dhawan A, Abdel-Latif M, Bajuk B, Stack J, and Lui K Intraventricular Hemorrhage and Neurodevelopmental Outcomes in Extreme Preterm Infants. Pediatrics 2013;133:55-62. [PubMed: 24379238]

17. Jelin Angie, Cheng Yvonne, and Caughey Aaron. 332:Necrotizing Enterocolitis and Risk of Infant Death. American Journal of Obstetrics and Gynecology 2012;206:S156

18. Kimberlin Debora F., Hauth John C., Owen John, Bottoms Sidney F., Iams Jay D., Mercer Brian M., Thom Elizabeth A., Moawad Atef H., Vandorsten J.peter, and Thurnau Gary R. Indicated versus Spontaneous Preterm Delivery: An Evaluation of Neonatal Morbidity among Infants Weighing $\leq 1000$ Grams at Birth. American Journal of Obstetrics and Gynecology 1999;180: 68389. [PubMed: 10076148]

19. Chen Aimin, Feresu Shingairai A., and Barsoom Michael J. Heterogeneity of Preterm Birth Subtypes in Relation to Neonatal Death. Obstetrics \& Gynecology 2009;114: 516-22. [PubMed: 19701029] 
20. Barros Fernando C., and Del Pilar Vélez Maria. Temporal Trends of Preterm Birth Subtypes and Neonatal Outcomes. Obstetrics \& Gynecology 2006;107:1035-041. [PubMed: 16648408]

21. Savitz DA, Dole N, Herring AH, Kaczor D, Murphy J, Siega-Riz AM, et al. Should spontaneous and medically indicated preterm births be separated for studying aetiology? Paediatr Perinat Epidemiol 2005;19:97-105 [PubMed: 15787884]

22. Schoendorf KC, Branum AM. The use of United States vital statistics in perinatal and obstetric research. Am J Obstetric Gynecol. 2006;194:911-915

23. Lydon-Rochelle MT, Holt VL, Cardenas V, et al. The reporting of pre-existing maternal medical conditions and complications of pregnancy on birth certificates and in hospital discharge data. Am J Obstet Gynecol. 2005;193:125-134 [PubMed: 16021070]

24. Stout Molly J., Busam Rachell, Macones George A., and Tuuli Methodius G. Spontaneous and Indicated Preterm Birth Subtypes: Interobserver Agreement and Accuracy of Classification. American Journal of Obstetrics and Gynecology 2014;211: 530.e1-4 [PubMed: 24844852] 


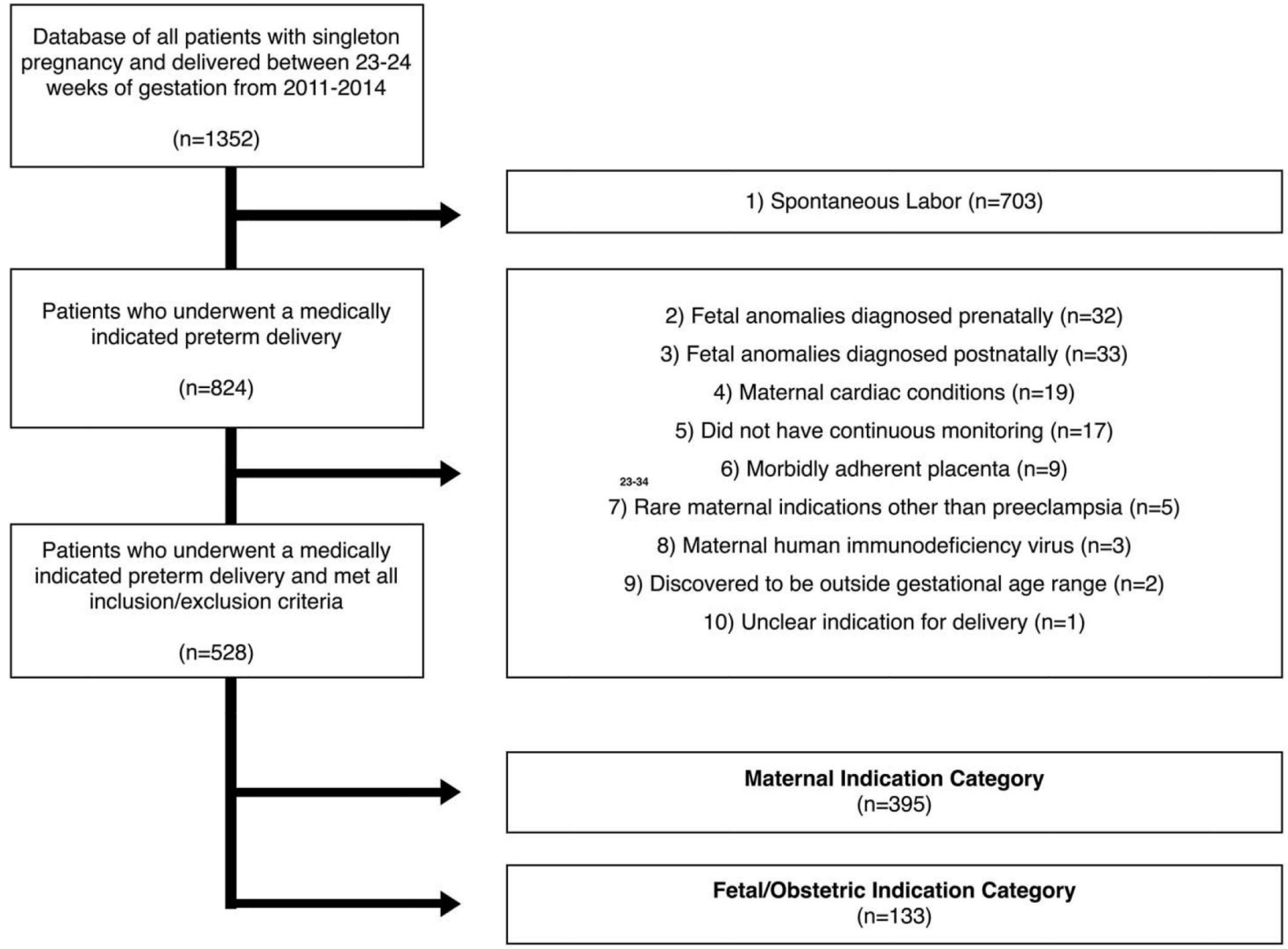

Figure 1.

Flow diagram of patients who underwent medically indicated preterm deliveries within our health system between 2011-2014 
Table 1.

Indications for Delivery

\begin{tabular}{|c|c|c|c|}
\hline \multicolumn{2}{|c|}{ Maternal $(n=395)$} & \multicolumn{2}{|l|}{ Fetal/Obstetric $(\mathbf{n}=\mathbf{1 3 3})$} \\
\hline Preeclampsia & $395(100 \%)$ & Non-Reassuring Fetal Status & $99(74.4 \%)$ \\
\hline & & Placental Abruption & $12(9.0 \%)$ \\
\hline & & Placenta Previa & $7(5.3 \%)$ \\
\hline & & Fetal Growth Restriction, Reversed End Diastolic Flow & $6(4.5 \%)$ \\
\hline & & Fetal Growth Restriction, Absent End Diastolic Flow & $5(3.8 \%)$ \\
\hline & & Oligohydramnios & $2(1.5 \%)$ \\
\hline & & Fetal Growth Restriction, Normal Umbilical Artery Dopplers & $1(0.8 \%)$ \\
\hline & & Suspected Isoimmunization & $1(0.8 \%)$ \\
\hline
\end{tabular}


Table 2.

Maternal Characteristics by Medical Indication for Delivery ${ }^{*}, \dot{t}$

\begin{tabular}{|c|c|c|c|}
\hline Maternal Demographic & Maternal Indication n=395 & Fetal/Obstetric Indication n=133 & $P$ value \\
\hline Age (yrs) & $27.4 \pm 6.1$ & $26.7 \pm 5.9$ & 0.27 \\
\hline Black & $195(49.4)$ & $75(56.4)$ & \multirow{4}{*}{0.41} \\
\hline White & $172(43.5)$ & $48(36.1)$ & \\
\hline Hispanic & $13(3.3 \%)$ & $6(4.5 \%)$ & \\
\hline Other & $15(3.8 \%)$ & $4(3.0 \%)$ & \\
\hline Government Insurance & $274(69.4 \%)$ & $100(75.2 \%)$ & 0.29 \\
\hline Married & $127(32.2 \%)$ & $39(29.3 \%)$ & 0.54 \\
\hline Nulliparous & $181(45.8 \%)$ & $52(39.1 \%)$ & 0.18 \\
\hline Previous vaginal delivery & $138(34.9 \%)$ & $58(43.6 \%)$ & 0.07 \\
\hline Previous Cesarean & $99(25.1)$ & $36(27.1)$ & 0.65 \\
\hline Previous Preterm Delivery & $108(27.3)$ & $43(32.33)$ & 0.27 \\
\hline BMI $\left(\mathrm{kg} / \mathrm{m}^{2}\right)$ & $35.1 \pm 8.5$ & $32.7 \pm 8.5$ & $<0.01$ \\
\hline Chronic Hypertension & $133(33.7 \%)$ & $26(19.6 \%)$ & $<0.01$ \\
\hline \multicolumn{4}{|l|}{ Diabetes } \\
\hline Pre-gestational Diabetes & $39(9.9 \%)$ & $11(8.3 \%)$ & \multirow{2}{*}{0.946} \\
\hline Gestational Diabetes & $21(5.3 \%)$ & $4(3.0 \%)$ & \\
\hline Renal Disease or Proteinuria & $14(3.5 \%)$ & $4(3.0 \%)$ & 0.77 \\
\hline Smoking & $57(14.4 \%)$ & $32(24.1 \%)$ & 0.03 \\
\hline Alcohol Use & $6(1.5 \%)$ & $3(2.3 \%)$ & 0.04 \\
\hline Drug Use & $22(5.6)$ & $9(6.8 \%)$ & 0.01 \\
\hline
\end{tabular}

* Medical Indications for delivery were categorized as (i) Maternal Indication (preeclampsia), (ii) Fetal/Obstetric Indication (growth restriction, non-reassuring fetal status, vaginal bleeding)

${ }^{\dagger}$ Values reported as a percent of patients in that primary indication category with the associated outcome or as mean \pm standard deviation 
Table 3.

Obstetric Characteristics by Medical Indication for Delivery

\begin{tabular}{|l|c|c|c|}
\hline \multicolumn{1}{|c|}{ Obstetric Characteristics } & Maternal Indication n=395 & $\begin{array}{c}\text { Fetal/Obstetric Indication } \\
\text { n=133 }\end{array}$ & P value \\
\hline Preeclampsia Diagnosed & $395(100 \%)$ & $47(35.3 \%)$ & $<0.01$ \\
\hline Estimated Fetal Weight (Most Recent Prior to Delivery) $(\mathrm{g})$ & $1368 \pm 531$ & $1049 \pm 546$ & $<0.01$ \\
\hline Fetal Growth Restriction Diagnosed & $56(14.2 \%)$ & $42(33.3 \%)$ & $<0.01$ \\
\hline $\begin{array}{l}\text { Critically Abnormal Umbilical Artery Dopplers (Absent or } \\
\text { Reversed) }\end{array}$ & $23 / 56(41.1 \%)$ & $32 / 43(74.4 \%)$ & $<0.01$ \\
\hline Antenatal Corticosteroids & & & \\
\hline None & $6(1.5 \%)$ & $10(7.5 \%)$ & $<0.01$ \\
\hline Partial & $56(14.0 \%)$ & $25(18.8 \%)$ & \\
\hline One Course & $316(80.0 \%)$ & $91(68.4 \%)$ & \\
\hline Repeat Course & $18(4.6 \%)$ & $7(5.3 \%)$ & 0.09 \\
\hline Male Fetus & $195(49.4 \%)$ & $77(57.9 \%)$ & $<0.01$ \\
\hline Birth Weight (g) & $1345 \pm 567$ & $1150 \pm 552$ & $<0.01$ \\
\hline Gestational Age at Delivery (weeks) & $30.4 \pm 2.8$ & $29.5 \pm 2.9$ & $<0.01$ \\
\hline Gestational Age at Delivery & & & $115(86.5)$ \\
\hline$\bullet 23-27.9$ weeks & $86(21.8 \%)$ & $40(30.1)$ & 0.10 \\
\hline$\bullet 28-31.9$ weeks & $164(41.6 \%)$ & $55(41.4)$ & \\
\hline$\bullet 32-34$ weeks & $144(36.6 \%)$ & $38(28.9)$ & \\
\hline Attempted Vaginal Delivery & $237(60.0$ & $15(40.5 \%)$ & \\
\hline Cesarean Delivery & & & $<$ \\
\hline
\end{tabular}


Table 4.

Neonatal Outcomes compared by Medical Indication for Delivery ${ }^{\mathscr{I}}$

\begin{tabular}{|c|c|c|c|c|}
\hline Neonatal Outcome & $\begin{array}{l}\text { Maternal Indication } \\
\quad \mathbf{n}=\mathbf{3 9 5}\end{array}$ & $\begin{array}{l}\text { Fetal/Obstetric Indication } \\
\quad \mathbf{n}=\mathbf{1 3 3}\end{array}$ & $\operatorname{AOR}(95 \% \mathrm{CI})$ & $P$ value \\
\hline Neonatal Composite Outcome & $85(21.5 \%)$ & $52(39.1 \%)$ & $1.9(1.14-3.21)^{*}$ & $<0.01$ \\
\hline Neonatal Death & $17(4.3 \%)$ & $13(9.8 \%)$ & $1.7(0.74-4.10)^{\dagger}$ & 0.02 \\
\hline Cord $\mathrm{pH}<7$ or base excess $<-12.5$ & $11(2.8 \%)$ & $17(12.8 \%)$ & $4.2(1.89-9.55)^{\hbar t}$ & $<0.01$ \\
\hline $\begin{array}{l}\text { Grade III-IV Intraventricular Hemorrhage } \\
\qquad \%)\end{array}$ & $15(3.8 \%)$ & $1(0.8 \%)$ & $0.1(0.02-1.13)^{t^{t}}$ & 0.08 \\
\hline Culture-Proven Sepsis & $29 / 371(7.8 \%)$ & $19 / 123(15.5 \%)$ & $1.7(0.84-3.46)^{t}$ & 0.01 \\
\hline Necrotizing Enterocolitis & $27(6.9 \%)$ & $14(10.6 \%)$ & $1.3(0.61-2.66)^{\dagger}$ & 0.17 \\
\hline 5-Minute Apgar $\leq 3$ & $26(6.6 \%)$ & $17(12.8 \%)$ & $1.4(0.68-2.89)^{* t}$ & 0.02 \\
\hline CPR in Delivery Room & $1(0.3 \%)$ & $1(0.8 \%)$ & - & 0.41 \\
\hline
\end{tabular}

* Adjusted for use of steroids, gestational age at delivery and BMI

${ }^{\dagger}$ Adjusted for use of steroids and gestational age at delivery

*Adjusted for use of steroids, gestational age at delivery and race

${ }^{q 1}$ Primary Neonatal Composite Outcome factors include the following: (i) death, (ii) $\mathrm{pH}<7$, (iii) 5 minute Apgar $\leq 3$, (iv) CPR in the labor and delivery room, (v) sepsis, (vi) IV. 